\title{
Aspectos laboratoriais ao diagnóstico da neurocisticercose: uma revisão bibliográfica
}

\section{Laboratory aspects to the diagnosis of neurocysticercosis: a bibliographic review}

\author{
Fernanda Oizimas Aguiar ${ }^{1}$ \\ Camila Moreira Leite $^{1}$ \\ Lucas Azevedo Dias ${ }^{1}$ \\ Vanessa D'Avila ${ }^{1}$ \\ Priscila Coutinho Agra ${ }^{1}$ \\ Luciana Costa ${ }^{1}$ \\ Marcello Mascarenhas ${ }^{1}$
}

\section{RESUMO}

Objetivo: Realizar uma revisão bibliográfica sobre as padronizações fundamentais do diagnóstico da neurocisticercose (NCC), frisando a importância da inclusão dos testes laboratoriais no padrão ouro. Métodos: 0 estudo trata-se de uma revisão bibliográfica da literatura. A elaboração deste estudo foi realizada seguindo as seguintes etapas: 1) identificação do tema e elaboração da questão norteadora; 2) pesquisa em banco de dados informatizados e na literatura; 3) tratamento dos dados coletados; 4) avaliação aprofundada dos materiais selecionados; 5) interpretação de resultados; 6) demonstração sintetizada do material compilado. Resultados: Através dessa revisão foi constatado que há casos em que a NCC é indetectável à imagem, sendo necessários exames complementares como testes laboratoriais e detecção do parasita por técnicas histopatológicas em material de biópsia de uma lesão cerebral ou medular. Os métodos laboratoriais são considerados de extrema importância e de baixo custo. Por isso os autores defendem no presente artigo a importância da inclusão dos testes laboratoriais ao diagnóstico da NCC no padrão ouro. Conclusão: Tendo em vista que os diversos fatores que são contributivos para que a endemia da NCC e da Teníase em nosso país e outros muito mais desenvolvidos, são principalmente hábitos simples de higiene e saneamento, cabe, então, aos governos e os órgãos responsáveis pela saúde e educação, um alto investimento em prevenção através da criação de programas de conscientização, para que se torne evidente a toda a população a gravidade do complexo teníase-NCC.

\section{PALAVRAS-CHAVE}

Neurocisticercose; Taeniasaginata; Taeniasolium; Elisa; Imunoblot.

\footnotetext{
${ }^{1}$ Centro Universitário Metodista - IPA. Artigo elaborado na disciplina de Projeto Interdisciplinar do Curso de Biomedicina.
} 


\section{ABSTRACT}

Objective: To carry out a bibliographic review on the fundamental standardizations of the diagnosis of neurocysticercosis (NCC), emphasizing the importance of including the laboratory tests in the gold standard. Methods: The study is a bibliographical review of the literature. The elaboration of this study was carried out following the following steps: 1) identification of the theme and elaboration of the guiding question; 2) research in computerized databases and literature; 3) treatment of collected data; 4) in-depth evaluation of selected materials; 5) interpretation of results; 6) synthesized demonstration of the compiled material. Results: Through this review it was observed that there are cases in which the NCC is undetectable to the image, requiring complementary tests such as laboratory tests and detection of the parasite by histopathological techniques in biopsy material of a brain or spinal cord injury. Laboratory methods are considered to be extremely important and inexpensive. Therefore, the authors defend in the present article the importance of the inclusion of the laboratory tests to the diagnosis of NCC in the gold standard. Conclusion: Since the various factors that contribute to the endemicity of NCC and Teniasis in our country and others that are much more developed are mainly simple hygiene and sanitation habits, it is then up to the governments and organs responsible for health and education, a high investment in prevention through the creation of awareness programs, so that the severity of the teniase-NCC complex becomes evident to the whole population.

\section{KEYWORDS}

Neurocysticercosis; Taeniasaginata; Taeniasolium; Elisa; Immunoblot. 


\section{INTRODUÇÃO}

A Neurocisticercose (NCC) é uma infecção causada pela infestação no sistema nervoso central (SNC) pelo embrião da Taeniasolium e é a principal forma clínica de apresentação da cisticercose. 0 ciclo biológico do complexo teníase-cisticercose envolve o ser humano como único hospedeiro do verme adulto denominado Taeniasolium, que, ao eliminar ovos nas fezes, contaminam o meio ambiente. 0 ser humano ao ingerir carnes suína e/ ou bovina com cisticercos (Cysticercuscellulosae) viáveis desenvolve a teníase. Foram descritos três estágios principais dos parasitas: vesicular (viáveis), coloidal (degenerativo) e calcificado (inativo) (BRASIL, 2015; ENSEÑATE et al, 2007).

Em todo o Mundo estima-se que cerca de 77 milhões de pessoas estejam infectadas pela Taeniasaginatae cerca de 2,5 milhões pela Taeniasolium. A incidência da neurocisticercose é proporcional à prevalência da teníase solium na população (AGAPEJEV, Svetlana; 2003). No Reino Unido a aquisição da doença após realização de viagens é a forma mais frequente, já que o país possui uma estrutura de saneamento exemplar. Na América do Norte, a NCC, durante muitas décadas, não recebeu muita atenção da comunidade científica, uma vez que era comum em comunidades de imigrantes, principalmente mexicanos e, em viajantes que voltaram de países endêmicos aos EUA. Porém, segundo dados de alta hospitalar na Amostra de Pacientes Nacionais de 2003 a 2012 constam estimadas 18.584 hospitalizações por neurocisticercose, mostrando que o aumento da incidência desta doença nos EUA é um fato, e que a doença se tornou um problema crescente de saúde pública (HUNTER et al, 2018; HALL et al, 2017;WINKLER, 2012; O'NEAL, Flecker, 2015).

No Brasil a prevalência da teníase soliumé da ordem de 2-3\%. As regiões com maior número de casos registrados são principalmente as regiões Sul e Sudeste, tanto em serviços de neurologia e neurocirurgia quanto em estudos anatomopatológicos. A baixa ocorrência de cisticercose em algumas áreas do Brasil, como por exemplo, nas regiões Norte e Nordeste, pode ser explicada pela falta de notificação ou porque o tratamento é realizado em grandes centros da sudeste, o que dificulta a identificação da procedência do local da infecção. 0 Ministério da Saúde registrou um total de 937 óbitos por cisticercose no período de 1980 a 1989, mas até o momento não existem dados disponíveis para que se possa definir a letalidade do agravo (BRASIL, 2005).

O diagnóstico da neurocisticercose (NCC) pode ser realizado pela demonstração conclusiva do cisticerco: detecção do parasita por técnicas histopatológicas em material de biópsia de uma lesão cerebral ou medular; visualização do escólex por tomografia computadorizada (TC) ou ressonância magnética (RM); e exame de fundo de olho, em casos de cisticercose intraocular. Na impossibilidade de demonstração direta do parasita, os testes laboratoriais e a análise em conjunto de dados clínicos e epidemiológicos permitem o diagnóstico da doença na maioria dos casos (BRASIL, 2005; ENSEÑAT et al, 2007).

Mesmo os exames de imagem como TC e RM sendo considerados padrão ouro no diagnóstico da NCC, existem casos não detectáveis por imagem. Além disso, o alto custo dessas tecnologias impede que muitos países endêmicos tenham acesso a esse tipo de diagnóstico e a um tratamento terapêutico adequado. Nestes casos os testes laboratoriais são essenciais para o diagnóstico da neurocisticercose(ENSEÑATE et al, 2007). Neste contexto, o artigo tem como objetivo realizar uma revisão bibliográfica sobre as padronizações fundamentais do diagnóstico da neurocisticercose (NCC), frisando a importância da inclusão dos testes laboratoriais no padrão ouro.

\section{MÉTODOS}

Utilizamos como objeto de pesquisa os sites de busca: Pubmed, Scielo e Lilacs, e auxílio do banco de dados da capes. Foram incluídos livros didáticos e os sites do ministério da saúde, OMS (Organização Mundial da Saúde) e ANVISA (Agência Nacional de Vigilância Sanitária). Usando os seguintes descritores: Neurocisticercose. Taeniasaginata.Taeniasolium. Elisa. Imunoblot.

$\mathrm{Na}$ busca de artigos, estudos, casos clínicos e publicações sobre o parasita TaeniaSolium e a neurocisticercose, estipulamos um período em torno de 20 anos, nos idiomas português, inglês e espanhol. Foram selecionados 50 artigos relacionados ao tema diagnóstico da neurocisticercose, dentre os quais 21 divergiam entre o assunto do resumo e introdução, 4 se baseavam em poucas referências e 2 não abordaram o assunto de maneira colaborativa a esse trabalho de revisão. As 23 fontes de 
revisão deste trabalho compreendem o período de publicação de até 20 anos e são relacionadas diretamente ao tema epidemiologia e diagnóstico da neurocisticercose e serviram como base de sustentação para essa pesquisa. Foram excluídos estudos sem resumo e não condizentes com a nossa busca.

Pesquisamos estudos referentes ao tema NCC, especificando padronizações fundamentais ao diagnóstico laboratorial. Conforme o aprofundamento do estudo, selecionamos os melhores artigos relacionados ao tema. Após, formamos os critérios para o seu desenvolvimento.

Para entender as características da pesquisa, é preciso, previamente, compreender todos os aspectos que englobam o ciclo biológico da Taeniasolium, estabelecer as diferenças entre teníase e cisticercose e traçar uma relação clínica- patológica da NCC.Em virtude da quantidade de definições encontrada na literatura científica, apresentamos algumas, consideradas relevantes para este estudo.

\section{RESULTADOS E DISCUSSÕES}

Nosso artigo consiste em uma revisão bibliográfica em busca dos métodos laboratoriais: Elisa (EnzymeLikedImmunoSorbetAssay) eImunoblot(WesternBlotting - WB), para ressaltar a importância da inclusão destes no padrão ouro.

0 método Elisa baseia-se em reações antígeno-anticorpo detectado por meio de reações enzimáticas (teste imunoenzimático) relacionadas a neurocisticercose. 0 Imunoblot é um teste bastante útil na detecção de antígenos. A escolha destes testes é devido a sensibilidade e a especificidade. Dos artigos selecionados 100\% usaram Ressonância Magnética (RM) e/ou Tomografia Computadorizada (TC) para realização do diagnóstico da neurocisticercose, tanto no Brasil, como no exterior.

A RM fundamenta-se em três etapas: alinhamento, excitação e detecção de radiofrequência. No alinhamento, por razões físicas e pela abundância, o núcleo de hidrogênio é o elemento utilizado para produzir imagens de seres biológicos. Assim, para que esses átomos sejam orientados numa certa direção, é necessário um campo magnético intenso ? habitualmente cerca de 1,5 Teslas. A etapa seguinte é a excitação. Sabe-se que cada núcleo de hidrogênio ?vibra? numa determinada frequência proporcional ao campo magnético em que está localiza- do. Assim, em 1,5 T, o hidrogênio tem frequência de 63,8 MHz. O aparelho emite então uma onda eletromagnética nessa mesma frequência. Existe uma transferência de energia da onda emitida pelo equipamento para os átomos de hidrogênio, fenômeno conhecido como ressonância (JUNIOR, 2001).

A TC consiste em uma fonte de raios-X que é acionada ao mesmo tempo em que realiza um movimento circular ao redor do paciente, emitindo um feixe de raios-X em forma de leque. No lado oposto a essa fonte, está localizada uma série de detectores que transformam a radiação em um sinal elétrico que é convertido em imagem digital. Dessa forma, as imagens correspondem a secções ("fatias") do crânio. A intensidade (brilho) reflete a absorção dos raios-X e pode ser medida em uma escala (unidades Hounsfield). (JUNIOR, 2001)

A TC e a RM são as principais ferramentas no diagnóstico da NCC e são tomados como o padrão-ouro, porém há casos em que a NCC é indetectável à imagem, sendo necessários exames complementares, como testes laboratoriais e detecção do parasita por técnicas histológicas em material de biópsia de uma lesão cerebral ou medular (CARPIO, 2002).

O Líquido cefalorraquidiano (LCR) é um dos principais fluídos do corpo humano e fornece informações primordiais em relação ao diagnóstico e acompanhamento de processos inflamatórios, infecciosos ou neoplásicos. 0 exame do LCR é o que fornece o maior número de elementos para detecção da neurocisticercose, devido à alteração no líquor pelos processos inflamatórios crônicos. Este exame se dá por punção lombar, que consiste na introdução de uma fina agulha entre os espaços vertebrais de L3/L4 ou L5/L6. A agulha precisa chegar no espaço subaracnóide, para que, com isso, seja coletado o LCR para posteriores análises (SILVA, 2004; GNUTZMANN et al, 2016; REY, 2008).

As alterações citoquímicas do LCR e a positividade das reações imunológicas para detecção de anticorpos anticisticerco, são importantes provas imunológicas. 0 ELISA é o mais utilizado, pois detecta anticorpos das classes IgG e IgM, obtendo-se uma especificidade de 95\% (VERONESI, 2011).

Os exames laboratoriais foram utilizados sempre como forma de diagnóstico complementar, e se mostrou presente em cerca de 70\% dos casos. Segundo Takayanagui, o desenvolvimento, nas últimas décadas, de téc- 
nicas imunológicas alternativas como as reações de imunofluorescência, hemaglutinação, imunoenzimáticas (ELISA - enzyme-linkedimmunosorbentassay) e blotting com glicoproteínas purificadas (EITB - enzyme-linkedimmunotransferblot) propiciou maior precisão do LCR no estabelecimento do diagnóstico.

O diagnóstico definitivo da infecção somente pode ser firmado pela pesquisa direta, demonstrando o parasito através dos exames anatomopatológicos das biópsias, necrópsias e intervenções cirúrgica (ALVERNE Passos Barbosa 2000). 0 eletroencefalograma (EEG) mostra baixa relação com os sintomas clínicos e com as lesões de NCC à neuroimagem (CARPIO, 2002).

Com base no que explicam os autores, fica clara a necessidade da inclusão dos exames laboratoriais nos protocolos de padrão ouro para o diagnóstico da NCC, dado sua precisão e custo inferior aos exames por imagem.

\section{CONCLUSÃO}

Na maioria dos Estados brasileiros a ausência de notificações compulsórias aos órgãos responsáveis pela saúde pública e, inclusive, a pouca perspectiva da população de acesso aos exames laboratoriais ou de imagem do diagnóstico da NCC, tornam o estudo clínico muito limitado. Além do mais, os diagnósticos que são considerados padrão ouro dependem da localização e manifestações do parasito.

Tendo em vista que os diversos fatores que são contributivos para que a endemia da NCC e da Teníase em nosso país e em outros muito mais desenvolvidos, são principalmente hábitos simples de higiene e saneamento. Cabe, então, aos governos e os órgãos responsáveis pela saúde e educação, um alto investimento em prevenção através da criação de programas de conscientização, para que se torne evidente a toda a população a gravidade do complexo teníase-NCC.

Portanto, concluímos que os exames considerados padrão ouro, os de imagem, possuem um elevado custo. Frente a realidade econômica dos países endêmicos, os exames de diagnóstico laboratoriais são a opção mais viável monetariamente e sua precisão é reconhecida na literatura. 


\section{REFERÊNCIAS}

AGAPEJEV, Svetlana; 2003. Disponível em: <http://www.scielo.br/pdf/\%0D/anp/v61n3B/17302.pdf>. Acesso em: $01 / 10 / 2018$.

BARBOSA, Alverne Passos et al. Cisticercose: Fatores Relacionados a Interação Parasito-Hospedeiro, Diagnóstico e Soroprevalência. Revista de Patologia Tropical. Goiânia, v. 29, n. 1, p. 17-34, jan-jun. 2000. Disponível em: <https://repositorio.bc. ufg.br/xmlui/bitstream/handle/ri/11710/Artigo\%20-\%20 Alverne $\% 20 \% 20$ Passos $\% 20 \% 20 \% 20$ Barbosa $\% 20-\% 20$ 2000.pdf? sequence=5\&isAllowed=y $>$. Acesso em 22/09/18.

BITTENCOURT, Paulo César Trevisol; e outros. Neurocistecercose em pacientes internados por epilepsia na cidade de Chapecó, Santa Catarina. Revista Neurologia UFSC; Disponível em $<$ http: / / www.scielo.br/scielo.php?pid=S 0004 $-282 X 1998000100008 \&$ script $=$ sci_abstract\&tlng=pt $>$ Acesso em $15 / 11 / 2018$

BRASIL. Ministério da Saúde. Secretaria de Vigilância em Saúde. Doenças infecciosas e parasitárias: guia de bolso. 5. ed. Amp. Brasília: Ministério da Saúde, 2005.

CARPIO, A. 2002. Neurocysticercosis: anupdate. Lancet Infect Dis, v. 2, p. 751-762.

CHIMELLI, Leila; Adriana F. Lovalho; e outros. Neurocysticercosis: contribution of autopsies to consolidation of the compulsory notification in Ribeirão Preto-SP, Brazil; Arq. Neuro-Psiquiatr. v. 56, n.3, B São Paulo, Sept. 1998. Disponível em: $\underline{\text { http:// }}$ www.scielo.br/scielo.php?script=sci arttext\&pid=S0004$\underline{-282 \times 1998000400010}$

ENSEÑAT, J. et al. Dificultades diagnósticas y terapéuticasenlaneurocisticercosis: presentacion de 6 casos y revisión de la literatura. Neurocirugía, v. 18, n. 2, p. 101-110, 2007. Disponível em: <https://www.sciencedirect.com/science/article/pii/ S1130147307702944>. Acesso em 22/09/18.

FOYACA-SIBAT, Humberto et al. Accuracy of Serological Testing for the Diagnosis of Prevalent Neurocysticercosis in Outpatients with Epilepsy, Eastern Cape Province, South Africa. PLoSNegl Trop Dis, v. 3, n. 12, p. e562, 2009.Disponível em: $<$ https://journals.plos.org/plosntds/article?id=10.1371/ journal.pntd.0000562\#abstract1>. Acesso em 22/09/18.

GUIMARÃES, Ronald Rodrigues; e outros; Neurocisticercose: Atualização sobre uma antiga doença. Revista Neurociências 2010. Disponível em: <http://www.revistaneurociencias.com. br/edicoes/2010/RN1804/362\%20atualizacao.pdf >. Acesso em 18/11/2018.

GNUTZMANN, Laísa et al. Analysis o freferenc evaluesof cerebrospinal fluid; SBAC, Revista Brasileira de Análises clínicas, 2016; Disponível em: <http://www.rbac.org.br/artigos/ analise-dos-valores-de-referencia-do-liquido-cefalorraquidiano-48n-3/> Acesso em 04/12/2018.

HALL, Rebecca L. et al. Survey of Obstetrician-Gynecologists in the United States About Taeniasis and Cysticercosis. The American Journal of Tropical Medicine and Hygiene, v. 96, n. 1, p. 233-242, jan. 2017. Disponível em: <https://www.ncbi.nlm. nih.gov/pmc/articles/PMC5239700/>. Acesso em 22/09/18.

HUNTER, Ewan et al. Active neurocysticercosis at the Hospital for Tropical Diseases, London: a clinical case series. Transactions of The Royal Society of Tropical Medicine and Hygiene, vol. 112, n. 7, p. 326-334, July 2018. Disponível em: <https://www.ncbi.nlm.nih.gov/pubmed/29982795>. Acesso em $20 / 09 / 18$

Jornal de Ciências Biomédicas e Saúde. JCBS, v.2, n.1, p.3944, 2016 <https://www.saudefacthus.com/jcbs>. Acesso em $01 / 10 / 2018$

JUNIOR, Edson Amaro e Yamashita, Hélio. Aspectos básicos de tomografia computadorizada e ressonância magnética. Revista Brasileira de Psiquiatria, 2001. Disponível emhttp:// www.scielo.br/pdf/rbp/v23s1/5560.pdf> Acesso em $04 / 12 / 2018$

MARQUES, Fabrício. Parasita Dissimulado. Revista Pesquisa FAPESP; 2005; Disponível em: <http://revistapesquisa.fapesp.br/2005/05/01/parasita-dissimulado/> Acesso em $15 / 11 / 2018$

MARTINS, Gerson Roberto; Tako, Karine Vaccaro. Neurocisticercose: uma revisão bibliográfica. Revista Uninga, 2008. Disponível em: <http://revista.uninga.br/index.php/uninga/article/view/681>. Acesso em 10/11/2018.

MIZOGUCHI, Vinícius Marcus; e Outros; Análise dos motivos para a prática de indivíduos com deficiência em modalidades coletivas e individuais; Revista Ciência em Movimento, Vol. 19 2017. Disponível em: <https://www.metodista.br/revistas/ revistas-ipa/index.php/RS/article/view/415>. Acesso em $17 / 11 / 2018$

OLIVEIRA, Clóvis de. Aspectos clínicos da neurocisticercose humana e viabilidade de padronização de Teste de Elisa para seu imunodiagnóstico. 2009. 105f. Dissertação (Pós Graduação) ? a Universidade Federal de Minas Gerais, Belo Horizonte, 2009. Disponível em: < http://www.bibliotecadigital. ufmg.br/dspace/bitstream/handle/1843/BUBD-8AFP4E/ neuroci_ncias__disserta__o_de_mestrado.pdf?sequence $=1>$ Acesso em 22/09/18

O'NEAL, Seth E., FLECKER, Robert H. Hospitalization Frequency and Charges for Neurocysticercosis. United States, 20032012. Emerging Infectious Diseases, v. 21, n. 6, p. 969-976, Jun. 2015. Disponível em: <https://www.ncbi.nlm.nih.gov/ pmc/articles/PMC4451927/>. Acesso em 22/09/18. 
TAKAYANAGUI, Oswaldo M, e Leite, João P. Neurocisticercose. Revista Sociedade Brasileira de Medicina Tropical, 2001. Disponível em <http://www.scielo.br/pdf/rsbmt/ v34n3/4998.pdf> Acesso em 15/11/2018.

UECKER, Marilei et al. Infecções parasitárias: diagnóstico imunológico de entero parasitoses. Revista Brasileira de Análises Clínicas (RBAC), Rio de Janeiro, v. 39, n. 1, p. 15-19, 2007 Disponível em: <http://files.saudecoletivaufrgs2013-1.webnode.com/200000623-5b30f5d246/Infec\%C3\%A7\%C3\% B5es\%20Parasit\%C3\%A1rias.pdf>. Acesso em 22/09/18.
WINKLER, Andrea Sylvia. Neurocysticercosis in sub-Saharan Africa: a review of prevalence, clinical characteristics, diagnosis, and management. Pathogens and Global Health, v. 106, n. 5, p. 261-74, Sep. 2012. Disponível em: <https://www.ncbi. nlm.nih.gov/pmc/articles/PMC 4005109/>. Acesso em 21/09/18.

ZAMMARCHI, Lorenzo et al. Epidemiology and Management of Cysticercosis and Taenia SoliumTaeniasis in Europe. Systematic Review 1990-2011. PLoS ONE, v. 8, n. 7, p. e69537, July 2013. Disponível em: <https://www.ncbi.nlm.nih.gov/pmc/ articles/PMC3726635/>. Acesso em 22/09/18. 